\title{
Experimenting with solidarity in biomedicine: from practice to principle?
}

\section{Luca Chiapperino and Francesco Panese}

In her contribution, Barbara Prainsack invites us to reflect upon often-neglected facets of contemporary visions of personalized medicine, which emphasize less the power of genomics to give room to other, non-genetic information with potentially higher levels of actionability. From social media data, to wearable sensors, or even consumption patterns, the path towards data-intensive, individualized medicine is not only paved by the explanatory promises of molecular and systems biology, but heavily draws upon categories of data traditionally not associated to medicine, which could yet prove fundamental in the development of monitoring and prevention strategies. As the author rightly points out - also through the mobilization of the narrative scenario of the novel The Circle - the future depicted by the use of these data is no less controversial than the one in which genomics plays the role of the "soloist" (p.4).

In particular, two assumptions behind big data medicine catch the concerned reflection of Prainsack. On the one hand, what could be called the moral suasion of citizens with regard to their alleged duties to collect and share more data, according to which those who do not contribute with their own data should actually be blamed of hampering the achievement of the societal good of a better medicine. In the author's view, this line of reasoning is fundamentally flawed in that it puts on a par the responsibilities and accountability of those who would actually control data (e.g. biobanks, public agencies, hospitals, or even private companies), and those who instead could both benefit and be harmed by their use. A patient and a network of biobanks pooling data, a citizen and a company marketing genetic testing direct-to-consumer - Prainsack argues - are social actors whose duties and obligations with regard to the sharing and controlling of data are not the same. Thus, any general call of duty to share and made accessible health data should take into consideration the imbalances of power currently existing in medicine, and devise effective collective strategies to protect the privacy of individual citizens. On the other hand, Prainsack's critique goes at the techno-epistemic core of the big data paradigm in biomedicine. The urge to share data in the discursive representations of personalized medicine often brushes over the concrete challenges of making data interoperable, and over the potential gains in terms of medically actionable information that could be obtained from data integration. Little is known, to this moment, about whether connecting heterogeneous datasets in medicine is an effort worth the huge investments it demands, or about the effective meaning and clinical benefits that could be obtained through this process.

Starting from this two-fold critique of personalized medicine, Prainsack argues for the need to innovate democratically this paradigm through social and political developments grounded on the normative framework of solidarity. In the understanding of its proponents (Prainsack and Buyx 2011), 
solidarity can be defined as the "shared practices reflecting a collective commitment to carry 'costs' (financial, social, emotional or otherwise) to assist others". Solidaristic acts and practices are, in other words, not only individual expressions of sympathy towards others' vulnerability in which we recognize our own. Quite the contrary, solidarity often manifests itself in the form of solidified social norms and structures (e.g. welfare systems and social welfare arrangements) that represent an explicit societal commitment to assist others at a nominal cost for each member of a society. For this reason, this concept may prove - according to the author - useful to account for the social and political challenges raised by personalized medicine. First, normative exercises of the solidarity principle could point to the need of bringing the personal and collective levels together in the context of big data medicine, by pointing to the fact that self-interested and other-regarding actions are indeed not mutually exclusive in this context (data that are useful to one patient can be useful also for the conditions afflicting another). Second, solidarity may highlight the multiple ways in which sharing one's data constitutes also a contribution to the achievement of collective goods. Third, solidaritybased approaches could also remind us that stratification should not lead to undue exclusion and should instead be driven by distributive considerations of benefit-sharing. Fourth, another important consideration stemming from the principle of solidarity has to do with questions of access, and the imperative to ensure that people facing projects of big data medicine are not left alone should they decide not, or be unable to partake to these endeavours (Prainsack and Buyx 2017).

In our commentary, we focus on both the moral and the epistemic aspects of the critique presented by Prainsack, and complement her proposal with an emphasis on the relevance and value of practical experimentations with solidarity in personalized medicine that are both moral and technoepistemic. As to the former, our objective is to complement her diagnosis on (what could be called) the moral economy (Fassin 2009) of personalized medicine with a reflection upon the role of solidarity-based engagements and moral practices to counter the dystopia furthered through this paradigm. As to the latter, instead, our goal is to show how working towards interoperability of data responds to distinct epistemic and technoscientific objectives of biomedical actors, some of which can arguably be characterized as going in the direction of furthering solidarity-sensitive practices in our societies through personalized medicine.

\section{Moral economies are unstable systems}

In the book At the Heart of the State, Didier Fassin and his colleagues explore what they call the moral world of institutions (Fassin et al. 2015). Drawing from ethnographic studies of different institutional settings - from courts, to the police, to employment centres - the authors present us with vivid reportages of the lived experiences and moral functioning of various services in our societies. Following the work of practitioners in these different contexts allows them revealing how institutions 
are "the site where the state is produced" (p.6); namely, how the "macro-sociological and the microsociological levels" (p.7) of state apparatuses are held together through the daily work, moral engagements, negotiations and relations of individual and collective agents working (for) them. Institutions are, in other words, the locus where the structure of a civic, legislative and regulatory framework unfolds into and is (re)produced by habits, judgements, sentiments, punishments - in one word, morals - of individual and collective subjects. In order to capture these two intertwining levels of institution-making the authors employ a distinction between moral economies and moral subjectivities. The former designate "the production, circulation and appropriation of values and affects regarding a given social issue" in a specific polity, at a given historical moment (Fassin et al. 2015, p.9; Fassin 2009). Roughly put, they constitute a common substantive understanding of the place, role and evaluation of actors gravitating around a given social issue: they provide general institutional positionings, they orient public sentiments and attitudes, and instruct individual and collective judgements of the situation. Moral subjectivities, by contrast, are instead the "processes by which individuals develop ethical practices in their relationships with themselves or others" (Fassin et al. 2015, p.9). They pertain to the level of the moral work involving individuals and collectives at the micro-level of routine activities in institutional settings, and typically consist of the moral tensions in the practice of turning the injunctions stemming from substantive representations of an issue (i.e. its moral economy) into embodied agency.

In this respect, moral subjectivities illustrate the lived body of a moral system: individual hesitations and pondering are the vivid solidification of the moral complexity of a situation, often neglected in its discursive and institutional representation. At the same time, however, moral subjectivities reveal also how agency is irreducible to the economy of sentiments, norms and principles giving meaning and value to a social phenomenon. The autonomy of agents to deviate from their professional ethos in order to lend a gesture of care, the decision to enforce more softly a given rule in the face of a particular situation, all testify of the generative power of subjective motivations, sentiments and relations in the face of an existing moral economy. In doing so, their book offers an in-depth demonstration of how moral economies are fundamentally unstable systems. Although providing the substantive framing of a certain matter in our societies, their existence in the social space necessitates the composing, decomposing and recomposing of their tenets by the concerned actors. This recognition, we argue, offers the opportunity for at least two reflections concerning Prainsack's socio-political uptake of the nascent paradigm of personalized medicine.

First, acknowledging the complex morality of institutional functioning suggests that although the concerns expressed by the author about personalized medicine are cogent - they belong to its moral economy, thus leaving open the possibility that relations, duties, care and reciprocal 
obligations in given settings and situations may be otherwise. This is not meant to deny that the idea behind this data-intensive type of medicine can arguably be characterized as constituting a depersonalization of medicine, reducing the individual patient to a mere digital representation stripped of its complex social belonging and existential complexity (Zwart 2016). Nor to mitigate the parallel critique showing how personalization as coveted medical reality often goes hand in hand with a problematic rhetoric of empowerment conceiving a devolution of responsibility to the citizenry with regard to their health (Juengst, Flatt, and Settersten 2012; cf. Prainsack 2017). Nor, finally, it is an exercise of wishful thinking denying the importance of hypes, programmatic visions, soft regulations, policy visions and biomedical in the practical unfolding of innovation (Hedgecoe 2004). Rather, what we mean here is to highlight the gap between some claims made to support the vision of personalized medicine and the complexities faced by the subjectivities working on the transformation of personalization into a reality of medicine. If we hold this distinction, we can see that the future is not given, that the problematic social circulation of particular technologies of monitoring and data brokering is not inevitable, that the moral fabric of personalized medicine is, in large part, still to be made. By holding a methodological distinction between moral economies and subjecties, we do not have to accept the dystopic version of personalised medicine criticized by Prainsack, mostly because the realization of this paradigm is still open, is still undecided: too many competing values, sentiments, desires, expectations (are likely to) play a fundamental role in the practical configurations this vision of medicine is going to engender.

Against this backdrop, Prainsack's work constitutes an important starting point. Her call for solidarity-based arrangements that could innovate democratically the domain of healthcare has the merit of highlighting alternative views of the moral economy of personalized medicine, which could instruct regulations genuinely serving the interests of public health. Yet, these views alone run the risk of obliterating the subjectivities currently experimenting with the moral economy of personalized medicine, which - as we have seen in the example of Fassin and his colleagues - cannot be reduced to the simple reproduction of the moral suasions and concealed disempowerment of citizens with regard to their health detectable at a discursive level. The 'moral world of personalized medicine' if we wanted to paraphrase these authors - may rather be defined by the substantive tension between generative practices and structuring discourses.

This leads us to a second reflection, which pertains to the strategies to further Prainsack's normative proposal in the nascent socio-political configurations of future medicine. What role could solidaristic practices that may be currently part of the daily, mundane work of experimenting with the paradigm of personalized medicine play in the promotion of her solidarity-based moral economy of future healthcare? As shown by (Hoeyer, Tupasela, and Rasmussen 2017) in the context of an 
international collaborative project, the material and informational flow of biomedical research often demands a type of moral engagement on the side of researchers that goes beyond the principles and regulations norming their professional practice. While the latter, as the authors show, are very useful to inform researchers about what they are allowed to do, it is rather through the "morality built into ties between people" (Hoeyer, Tupasela, and Rasmussen 2017, p.393) that research collaborations among scientists and donors are kept flowing. This recognition goes in the direction of the distinction we have drawn from Fassin and colleagues between moral economies and subjectivities, and suggests that the same may currently be happening with regard to solidarity. Regardless of whether this principle is integral or foreign to the moral economy of personalized in a given socio-political setting, solidarity could be seen - in this vein - as one among other values that is being negotiated in the daily subjective work of researchers experimenting with personalization. And, indeed, some evidence to support this view may be found in the literature. From the decision to implement institutional policies for data restitution in genomic research that take into consideration vulnerable (biological) relatives of sample donors although not required by current legislation (Hens et al. 2011), to the development of alternative models of informed consent that deviate from the gold standard of general consent to give more room to patient participation and partnerships between them and the researchers (Blasimme and Vayena 2016), we have reasons to think that solidaristic practices are already part and parcel of the moral world of personalized medicine. This posits the opportunity of a thoroughly complementary strategy for the promotion of Prainsack's proposal, and one that methodologically starts from a problematization of the hiatus between the complexity of moral practices and the economies of values that guide biomedicine. Ultimately, the importance of promoting solidarity-based arrangements in personalized medicine is a strong political argument for Prainsack, which - at the same time - may be a very practical concern for researchers and practitioners currently working on the translation of personalization into a medical reality. What if, then, we needed more to generalize moral subjective experimentations with solidarity than to promote it as a principle in personalized medicine? To rephrase Bernard Williams' critique of Kantian moral philosophy, future patients may need the benefits of human gestures of solidarity, not the benefits of its principle (Williams 1999).

\section{The epistemic work towards interoperability and solidarity}

The same kind of reasoning could apply to the epistemic and technoscientific fabric of personalized medicine, and consequently to the critique Prainsack moves to the epistemic value of interoperability in personalized medicine. The visions of data-intensive, individualized medicine discussed by the author are in fact currently being translated into concrete technoscientific assemblages that are pivotal to the establishment of personalized medicine as translational research and biomedical practice in a given socio-political setting. In the Swiss case (Meier-Abt et al. 2015), for instance, we can also 
underline the discursive investment of interoperable data infrastructures ((Swiss Personalized Health Network 2017) with a hyped enthusiasm. Building an infrastructure of interoperable datasets is perhaps the main declared objective of Swiss initiatives, which is presented as capable of allowing "the molecular analysis of samples, the sharing of data for a large population, the development of standardized bioinformatics tools [...] and finally, the interpretation of these data enabling optimal therapeutic decision-making" (Coukos et al. 2014, p.1099). Yet, a closer look at the different research, translational and clinical endeavours involving actors in the Swiss biomedical community reveals how interoperable data infrastructures - more than constituting an object - should be regarded as an objective (cf. Guchet 2016) towards which these actors work by negotiating its experimental, technological, social, economic, legal and political facets. A few examples may be useful here to illustrate how interoperability of data in the infrastructures at the centre of this and similar endeavours constitute concrete elements of professional work towards the alignment of various technoscientific promises with medical reality.

Driven by imperatives of research discoveries and the development of innovative therapeutics or biomarkers, those who have developed platforms of biobanking for sample storage and sharing (Mooser and Currat 2014) consider the integration of data as a fundamental resource to harness the potentialities of current biomedical technologies, and to streamline the research process of development for new preventive and therapeutic strategies. Without going as far as considering it capable to "yield meaningful, medically actionable information" (p.8) - something that Prainsack arguably underlines as being unwarranted - practicing the interoperability of patient health records and data produced through the analysis of biobanking samples is in this context building an environment for research towards personalized medicine. Interoperability means here establishing a convention of practices and norms (e.g. common ontology of different data, homogeneous consent standards, etc.) among citizen-donors and the community of clinician researchers. Differently from this example, interoperability is instead a matter of hermeneutics and interpretation for those working toward the development of bioinformatics tools for data analysis (Lescuyer, Hochstrasser, and Rabilloud 2007; Sansone et al. 2012). Rather than pointing to the relational function of interoperable biological data, in this concrete engagement with data integration what is at stake is the combination of different layers of biological data in a meaningful way. Bioinformatic interoperability of multiomic data - but also digital health data collected through technologies such as trackers, sensors, or wearables (see (Salathé et al. 2012; Lovis and James 2016; Trono 2016) - finds its rationale into the capacity to describe systems of interaction between biological phenomena of different nature. In this respect, the promises attached to the operationalization of interoperability seem to sketch a dynamic that is symmetrically different from the one we encountered in the example above. While in the first 
case interoperability operates as an invisible, background feature of the research ecology pivoting around biobanking, here interoperable datasets are rather supposed to resurface systematically - or even automatically, if we take at face value the promissory rhetoric attached to artificial intelligence (Howard and Huber 2016) - as an informed grasp of the relevant property of biological systems and their variation from a statistical average.

Needless to say, the questions of medical actionability of this information emphasized by Prainsack are yet another layer of complexity in the different pragmatic engagements with data interoperability we have briefly illustrated through the Swiss cases. However, what the examples above show is that building infrastructures for data-driven medicine is a layered, complex, and heterogeneous practice, which is informed by distinct epistemic and technoscientific ends, as well as visions as to why it is worth producing, pooling and implementing knowledge for the personalization of future medicine. A process that, as we have seen, gives shape to distinct pragmatic functions of interoperability, and that, we might add, is also likely to be thoroughly imbued with the subjective experimentations with the moral economy of personalized medicine highlighted in the previous section. This is the next (and last) piece of the jigsaw portrayed by Prainsack upon which we wish to elaborate.

The epistemic engagements with interoperability and sharing of data can indeed be characterized also as a plurality of normative and value-laden engagements with the socio-political configurations of personalized medicine. Building an infrastructure of interoperable data is, in this vein, not just creating an ecosystem allowing researchers, patients and clinicians to pursue different epistemic objectives, but also an exercise of co-construction of the moral life of such infrastructures. One example - always drawn from our observations in the Swiss context - can illustrate these moments of co-production (Jasanoff 2004) in personalized medicine: the attempt to provide technical solutions to the ethical issues of privacy and re-identification in IT infrastructures of personalized data sharing. Current configurations of research, and the public debate ('Génome chiffré, génome protégé' 2017) around Swiss initiatives towards personalized health partly revolve in fact around the relevance and utility of strategies for the encryption of the data collected. Developing effective cryptography standards is, in this context, not just an engineering problem for the construction of informatics systems of data-sharing (Check Hayden 2015), but rather constitutes the opportunity to infuse the machinery of personalized medicine with values of "digital trust" and privacy-conscious technological standards (Barraud 2017). Striking the right balance between circulation of different data, minimization of risks for re-identification and prevention of breaches of access through $a$ technical standard for data handling and sharing is, in other words, a way to promote trusted participation in this endeavour, and to prevent indiscreet access to these data by unwarranted actors. 
Specifically, the interests of insurance companies and the reported cyber attacks to which healthcare institutions are subjected in the US ('Health Care Industry Cybersecurity Task Force - PHE' 2018) provide, in the case at issue, a moral justification for developing robust encryption standards in interoperable data systems. In brief, pursuing the techno-epistemic objective of a secure and interoperable data infrastructure can reasonably be characterized as one facet of the moral work towards an equitable and privacy-promoting configuration of personalized medicine.

The example of encryption shows therefore how developing interoperable data infrastructures means also aligning techno-epistemic standards with values of trust in the healthcare system and with the political struggles to prevent potential discrimination of patients and citizens. By ensuring that any illegitimate user of health data is kept out of the infrastructure, standards of encryption represent a resource for biomedical actors to sketch a specific social circulation of their work towards personalized medicine. And one, we argue, that could go in the direction of rendering solidaristic practices both a moral and a technoscientific condition of possibility of personalized medicine. The normative and technical imperative of cryptography could in fact arguably be seen as no less than one - limited, yet significant - strategy to ensure that openness of health data in the future of medicine does not undermine existing solidaristic structures in healthcare. In this respect, Prainsack's critique of interoperability and data sharing in these endeavours is an important reminder of how "a critical examination of the assumptions and consequences of such an approach in medicine is an important task" (p.9). Yet, what our analysis has tried to show is that interoperability may not only be valuable to a pre-given and static rhetoric worth her critiques, but also may qualify as a symmetric and complementary practical engagement with the moral filigree of data-intensive and personalized medicine. Weaving the sociotechnical fabric of personalization in medicine does not rest solely on the problematic economy of values, norms and sentiments criticized by Prainsack. Rather, it is a practical process straddling the social and techno-epistemic value of this endeavour, and one that in at least one telling example - displays material and technological experimentations that could be compatible with her call to promote solidarity in the social structure and power dynamics of medicine.

\section{Conclusions}

The contribution by Barbara Prainsack invites us to reflect upon the socio-political innovations that the normative exercises of solidarity could bring into the realization of personalized medicine. In the present commentary, we we have highlighted multiple ways in which solidaristic practices can already be observed in the unfolding of personalization in medicine both at the moral and epistemic level. In this respect, our analysis can be regarded as a complement to Prainsack's critique of the moral economy of data-intensive and personalized medicine. Specifically, our work constitutes an invitation to consider the framework proposed by the author not just as a positive normative guidance 
in the societal appraisal of socio-political initiatives towards personalized medicine. Rather, our focus on micro-level experimentations with personalization reveals how solidarity thinking qualifies also as productive methodological operator allowing to reconstruct how, amidst the yet indeterminate configurations of future medicine, its value is being negotiated in, and thus could flourish from the praxis of making personalized medicine a social reality. 


\section{Bibliography}

1. Barraud, Emmanuel. 2017. 'EPFL Teams up with Partner Organizations to Build "digital Trust”, December. https://actu.epfl.ch/news/epfl-teams-up-with-partner-organizations-to-build-/.

2. Blasimme, Alessandro, and Effy Vayena. 2016. 'Becoming Partners, Retaining Autonomy: Ethical Considerations on the Development of Precision Medicine'. BMC Medical Ethics 17 (November): 67. https://doi.org/10.1186/s12910-016-0149-6.

3. Check Hayden, Erika. 2015. 'Extreme Cryptography Paves Way to Personalized Medicine'. Nature News 519 (7544): 400. https://doi.org/10.1038/519400a.

4. Coukos, George, Olivier Michielin, Pierre-Yves Dietrich, and Matti Aapro. 2014. 'Ensemble Vers L’oncologie de Précision !' Revue Médicale Suisse, no. 431: 1099-1100.

5. Fassin, Didier. 2009. 'Moral Economies Revisited'. Annales. Histoire, Sciences Sociales 64th Year (6): 1237-66.

6. Fassin, Didier, Yasmine Bouagga, Isabelle Coutant, Jean-Sebastien Eideliam, and Fabrice Fernandez. 2015. At the Heart of the State: The Moral World of Institutions. London: Pluto Pr.

7. 'Génome chiffré, génome protégé'. 2017. 24 April 2017. https://www.letemps.ch/sciences/genome-chiffre-genome-protege.

8. Guchet, Xavier. 2016. La Médecine Personnalisée. Un Essaie Philosophique. Médecine et Sciences Humaines. Les Belles Lettres. https://www.lesbelleslettres.com/livre/1277-lamedecine-personnalisee.

9. 'Health Care Industry Cybersecurity Task Force - PHE'. 2018. Accessed 1 March 2018. https://www.phe.gov/preparedness/planning/CyberTF/Pages/default.aspx.

10. Hedgecoe, Adam. 2004. The Politics of Personalised Medicine: Pharmacogenetics in the Clinic. New York: Cambridge University Press. http://search.ebscohost.com/login.aspx?direct=true \&scope=site \&db=nlebk\&db=nlabk\&AN=16 5311.

11. Hens, Kristien, Herman Nys, Jean-Jacques Cassiman, and Kris Dierickx. 2011. 'The Return of Individual Research Findings in Paediatric Genetic Research'. Journal of Medical Ethics 37 (3): 179-83. https://doi.org/10.1136/jme.2010.037473.

12. Hoeyer, Klaus, Aaro Tupasela, and Malene Bøgehus Rasmussen. 2017. 'Ethics Policies and Ethics Work in Cross-National Genetic Research and Data Sharing: Flows, Nonflows, and Overflows'. Science, Technology, \& Human Values 42 (3): 381-404. https://doi.org/10.1177/0162243916674321.

13. Howard, Paul, and Peter Huber. 2016. Unlocking Precision Medicine. New York: Encounter Books. 
14. Jasanoff, Sheila. 2004. States of Knowledge: The Co-Production of Science and the Social Order. Routledge.

15. Juengst, Eric T., Michael A. Flatt, and Richard A. Settersten. 2012. 'Personalized Genomic Medicine and the Rhetoric of Empowerment'. The Hastings Center Report 42 (5): 34-40. https://doi.org/10.1002/hast.65.

16. Lescuyer, Pierre, Denis Hochstrasser, and Thierry Rabilloud. 2007. 'How Shall We Use the Proteomics Toolbox for Biomarker Discovery?' Journal of Proteome Research 6 (9): 3371-76. https://doi.org/10.1021/pr0702060.

17. Lovis, Christian, and Clara James. 2016. 'Santé Digitale: Petit Guide À L'usage Du Néophyte'. Revue Médicale Suisse 12 (521): 1108-1112.

18. Meier-Abt, Peter J., Ron Appel SIB, Urs Frey, Detlef Günther, Vincent Mooser, Nicole ProbstHensch, Michael Röthlisberger, and Daniel Vonder Mühll. 2015. 'Implementation of the "Swiss Personalized Health Network"(SPHN) Initiative'. https://www.sbfi.admin.ch/dam/sbfi/de/dokumente/report_implementationoftheswisspersonalize dhealthnetworksphninit.pdf.download.pdf/report_implementationoftheswisspersonalizedhealthn etworksphninit.pdf.

19. Mooser, V, and C Currat. 2014. 'The Lausanne Institutional Biobank: A New Resource to Catalyse Research in Personalised Medicine and Pharmaceutical Sciences'. Swiss Medical Weekly, December. https://doi.org/10.4414/smw.2014.14033.

20. Prainsack, Barbara. 2017. Personalized Medicine: Empowered Patients in the 21st Century? New York: NYU Press.

21. Prainsack, Barbara, and Alena Buyx. 2011. Solidarity: Reflections on an Emerging Concept in Bioethics. Citeseer.

22. _ 2017. 'Solidarity in Practice II: Personalised Medicine and Healthcare'. In Solidarity in Biomedicine and Beyond, 123-44. Cambridge: Cambridge University Press. http://ebooks.cambridge.org/ref/id/CBO9781139696593.

23. Salathé, Marcel, Linus Bengtsson, Todd J. Bodnar, Devon D. Brewer, John S. Brownstein, Caroline Buckee, Ellsworth M. Campbell, et al. 2012. 'Digital Epidemiology'. PLOS Computational Biology 8 (7): e1002616. https://doi.org/10.1371/journal.pcbi.1002616.

24. Sansone, Susanna-Assunta, Philippe Rocca-Serra, Dawn Field, Eamonn Maguire, Chris Taylor, Oliver Hofmann, Hong Fang, et al. 2012. 'Toward Interoperable Bioscience Data'. Nature Genetics 44 (2): 121-26. https://doi.org/10.1038/ng.1054. 
25. Swiss Personalized Health Network. 2017. 'Factsheet SPHN at a Glance'. http://www.samw.ch/dam/jcr:962d21ee-870e-4989-b81d71605b454980/factsheet_sams_sphn_2017.pdf.

26. Trono, Didier. 2016. 'Switzerland and the Digital Health Revolution'. CHIMIA International Journal for Chemistry 70 (12): 851-52. https://doi.org/10.2533/chimia.2016.851.

27. Williams, Bernard Arthur Owen. 1999. Problems of the Self: Philosophical Papers 1956 - 1972. Reprint. Cambridge: Cambridge Univ. Press.

28. Zwart, H. A. E. (Hub). 2016. 'The Obliteration of Life: Depersonalization and Disembodiment in the Terabyte Era'. New Genetics and Society 35 (1): 69-89. https://doi.org/10.1080/14636778.2016.1143770. 